\title{
MAGNETISM AND MAGNETORESISTANCE OF MULTILAYERS PREPARED ON MICROSTRUCTURED SUBSTRATES
}

\begin{abstract}
T. Shinjo, T. Ono, K. Shigeto and Y. Sugita*
Institute for Chemical Research, Kyoto University, Uji, Kyoto-fu 611, Japan

Magnetic multilayers with a new type of structural modification were prepared by ultrahigh vacuum deposition onto substrates with V-shaped microgrooves and used as the samples for the following two novel studies: One is the study of giant magnetoresistance in a new geometry, CAP, where the electric current is at a certain angle to interfaces. Enhancement of magnetoresistance in CAP geometry, in comparison to the normal current in-plane magnetoresistance, was confirmed. The other is the study of magnetic thin wires, which were prepared by depositing in a tilted direction to the surface. A preliminary result of magnetization reversal investigation is presented.
\end{abstract}

PACS numbers: 75.70.-i, 75.70.Pa, 85.30.Vw

\section{Introduction}

Microfabrication techniques have been developed in the field of semiconductor physics and utilized extensively for fundamental studies on mesoscopic systems and also for various devices. On metallic and magnetic systems also, there have been several pioneering works [1-3]. Studics on magnetic fine particles have a long history but until very recently size distributions are inevitable in actual samples. Micromagnetic systems, whose shape and size are regulated, are prepared by using microfabrication techniques and thus arguments with a quantitative comparison of experimental results and theories have been made possible. A recent attractive topic in the field of magnetism is the giant magnetoresistance (GMR) and as is described later, GMR in CPP geometry (current perpendicular to plane) is an example of subject for which the application of microfabrication techniques is efficient.

In the present paper, another approach is introduced. Microfabrication techniques are not applied for magnetic materials directly but microstructured surfaces are used as substrates for magnetic film depositions. On substrates with V-shaped microgrooves, multilayers for CAP-MR studies were prepared by depositing films in the direction perpendicular to the substrate surfaces. Magnetic multilayers with

*Present address: Central Research Laboratories, Matsushita Electric Ind. Co. Ltd. Seikacho, Kyoto-fu 619-02, Japan. 
a wire shape were prepared by depositing in a tilted direction. A preliminary experimental result to investigate the magnetization reversal phenomena in multilayer wires is described.

\section{V-shaped microgroove substrates}

V-shaped microgrooves are constructed by applying an anisotropic etching to micropatterned Si surfaces. The technique to prepare V-grooves is the well established one in the field of semiconductors [4]. The procedure is schematically illustrated in Fig. 1. A (100)-oriented $\mathrm{Si}$ wafer, whose surface is covered by a $\mathrm{SiO}_{2}$ layer, is masked by photoresistance and by electron beam lithography, a stripe pattern with alternating open and protected areas is constructed. The smallest period of stripes in the present samples was $0.5 \mu \mathrm{m}$, consisting of $0.1 \mu \mathrm{m}$ oxide

(i)

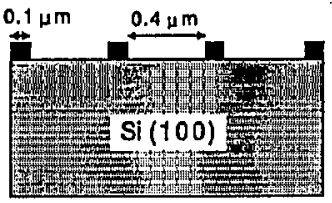

(ii)

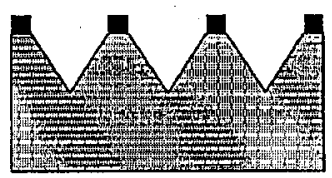

(iii)

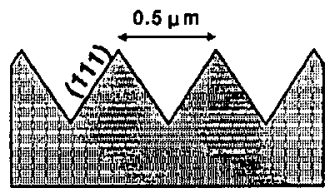

Fig. 1. Schematic illustration of preparation process of the V-groove microstructured substrates. (i) (100) $\mathrm{Si}$ wafer with microstripe pattern of alternating $\mathrm{SiO}_{2}$ and $\mathrm{Si}$ areas. (ii) Wet etching with $\mathrm{KOH}$ solution. (iii) V-groove.

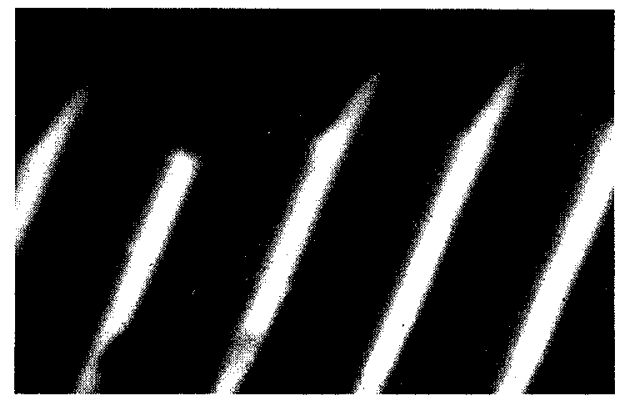

Fig. 2. SEM image of substrate surface. 
and $0.4 \mu \mathrm{m}$ Si layers. Micropatterned Si wafers were then dipped in KOH solution ( $20 \mathrm{w} \%)$ to etch the surface. Since the etching rate of (100) plane is 2 order faster than that of (111), the grooves with (111) planes are finally performed. A typical etching time length to produce a groove structure having sharp edges was 5 min. By scanning electron microscope (SEM), the achievement of V-groove structures on Si wafers was confirmed (Fig. 2). It was also confirmed that after multilayer deposition the surface structure of deposited film has not significantly changed from the original V-groove structure of the substrate.

\section{Non-coupled type GMR and geometry}

GMR means a large conductivity change associated with a transformation of magnetic structure, from antiparallel to parallel. In the case of the first example, $\mathrm{Fe} / \mathrm{Cr}$ multilayers [5], the interlayer antiferromagnetic coupling is the origin of antiparallel alignment. Afterwards, $\mathrm{Co} / \mathrm{Cu}$ multilayers were found to be another typical example of such a coupled type GMR system [6,7]. However, the interlayer coupling is not a necessary condition for GMR. By utilizing the difference of coercive forces, an antiparallel alignment may be induced in multilayers and the enhancement of resistance is realized, that is GMR in a non-coupled system. In 1990 the author's group prepared non-coupled multilayers with the structure of $[\mathrm{Cu} / \mathrm{Co} / \mathrm{Cu} / \mathrm{NiFe}] \times \mathrm{n}$ and found a significantly large MR ratios [8]. The IBM group prepared a non-coupled three layer sandwich system (spin-valve), which includes two NiFe layers: one is magnetically soft, while the other has a large coercive force because of the exchange anisotropy due to the coupling with an antiferromagnetic layer [9].

In contrast to coupled type GMR, the resistivity change of non-coupled type can be induced by a small magnetic field. For the purpose of technical applications, especially to use as magnetic recording heads, it is required that a large MR ratio is induced by a very small field. Non-coupled type multilayers have definite merits to realize such soft MR properties and actually spin-valve type recording heads are being tested.

Non-coupled type multilayers are useful also for basic studies. In coupled systems, GMR only appears at certain spacer layer thicknesses, where an antiferromagnetic interlayer coupling exists. In contrast, GMR of non-coupled systems is realized at any spacer layer thickness, as far as the interlayer coupling is negligible. Therefore, as is described in the next section, the dependence of MR on the spacer layer thickness can be checked continuously.

In almost all cases, magnetic films are prepared on substrates with two-dimensional shapes. It is very difficult to measure the CPP resistivity of a metallic film, because the resistivity is extremely small. However, novel properties of artificial multilayers may be associated with periodicity along the perpendicular direction. It is natural to expect a larger MR ratio in CPP geometry because electrons meet more interfaces. Recently, several pioneering works have been carried out to measure MR in CPP geometry (see Fig. 3). The first attempt to measure the CPP-MR has been made by Michigan group [10]. In order to measure the intrinsic, extremely small resistivity, a GMR multilayer was sandwiched by two superconducting $\mathrm{Nb}$ layers and those were used as the contact electrodes to eliminate trivial resistances 

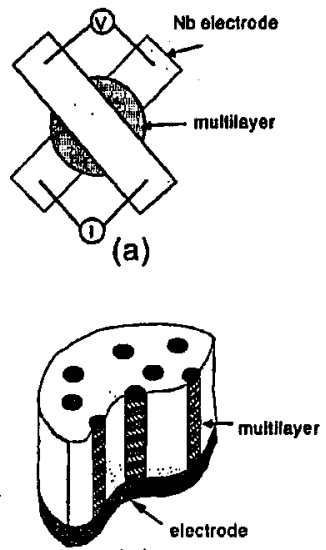

(c)

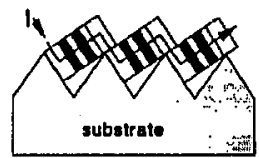

(e)

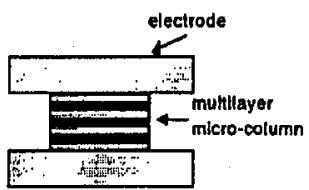

(b)

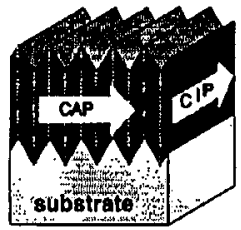

(d)

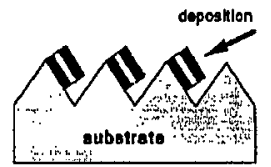

(f)

Fig. 3. Schematic illustration of sample structures for the following studies: (a) multilayer sandwiched by superconducting electrodes for CPP-MR studies; (b) microfabricated multilayer pillar for CPP-MR studies; (c) multilayer wires in nanoporous membrane prepared by electrodeposition for CPP-MR studies; (d) multilayer on V-groove for CAP-MR studies (deposited perpendicularly); (e) multilayer on V-groove for CPP-like MR studies (deposited in a tilted direction); (f) multilayer wires on $\mathrm{V}$-groove (deposited in a tilted direction).

and contributions from fractional currents in the plane. It was thus observed that the CPP-MR was significantly larger than the normal CIP-MR (current in-plane) in a simultaneously prepared sample. However, the measurement in this method was limited only at low temperatures because superconducting electrodes were used. Subsequently, Philips group has adopted a microfabrication technique to produce microcolumns of GMR multilayers [11]. The size of microcolumns was about $1 \mu \mathrm{m}$ but the height of the columns was not larger than the width. Namely the structure was not very suitable for the CPP-MR measurements. They also found the enhancement of MR in CPP geometry in comparison to CIP. A temperature dependence of MR can be measured in this case. However, it is not easy to apply the microfabrication techniques for metallic films. The third method was reported by the Leuven group [12]. They have used an electrodeposition method to prepare multilayers in small holes of membrane filtres and actually succeeded in making microwires of multilayers in each hole. The structure of sample is the ideal one for CPP-MP measurements and they observed fairly large MR ratios 
for $\mathrm{Co} / \mathrm{Cu}$ multilayer wires. However, concerning the choice of elements and the quality of multilayer structure, the feasibility of electrodeposition seems to be very limited.

The fourth method is to utilize microstructured substrates as is shown in the next section [13].

\section{CAP-MR}

Non-coupled type GMR multilayers were deposited on V-groove substrates in the normal direction to the original substrate surface (Fig. $3 \mathrm{~d}$ ). The constitution, for instance, is $[\mathrm{Cu}(580 \AA) / \mathrm{Co}(12 \AA) / \mathrm{Cu}(580 \AA) / \mathrm{NiFe}(12 \AA)] \times 34$. Figure $4 \mathrm{a}$ is magnetization vs. field curve of a sample at $5 \mathrm{~K}$. The curve has a two-step feature which indicates the exchange coupling between magnetic layers is negligible and an antiparallel alignment of adjacent layer magnetizations is accomplished in the course of field sweeping.

The MR curve is reproduced in Fig. 4b. The CAP-MR values are the resistivity values observed with a current perpendicular to the grooves. On the other hand, CIP-MR values are measured with a current parallel to the grooves. The magnetic field was applied parallel to the grooves in both measurements. If the current direction is perpendicular to the grooves, it is parallel to the original $\mathrm{Si}(100)$ basal plane but is at $54.7^{\circ}$ to the multilayer planes. The deposited multilayer has a sufficient thickness, $4 \mu \mathrm{m}$, to neglect a contribution from the wrinkled parts in the sample with a prismatic shape in upper and lower sides. This new geometry of MR

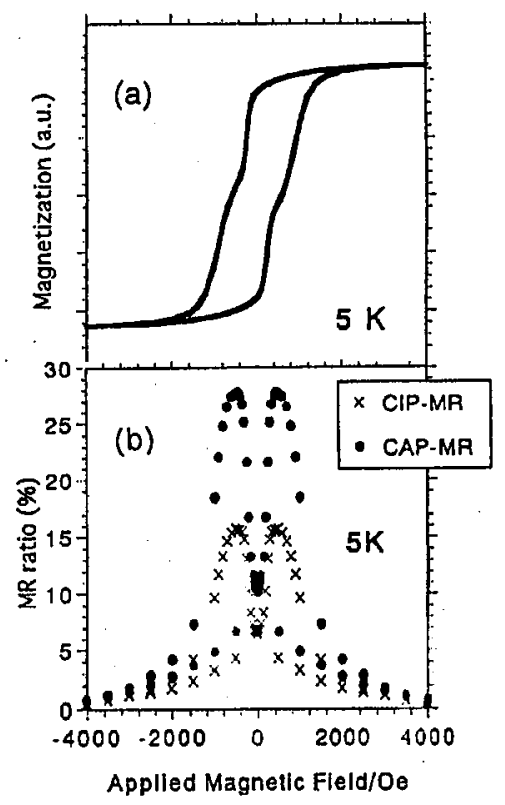

Fig. 4. (a) Magnetization curve at $5 \mathrm{~K}$ for a non-coupled GMR multilayer sample, the structure is described in the text; (b) MR values at $5 \mathrm{~K}$ for the same sample in CAP and CIP geometry. 
measurements was named CAP, with a current at an angle to plane [14], and apparently the observed CAP-MR ratio is larger than the simultaneously measured CIP-MR one.

Compared with the previous three CPP studies, the present CAP-MR measurements have some features. The current direction is not at $90^{\circ}$ but much lower, and in this sense the geometry is far from the ideal CPP. A merit is that together with CAP measurements, CIP also is possible for a sample simultaneously prepared in the same condition. Therefore, the CAP- and CIP-MR values are able to be obtained for the same sample. In addition, by changing the current direction in the original plane, the intermediate MR values between CIP and CAP measured continuously. By extrapolation using these values, The CPP-MR values are estimated. The theoretical background of this procedure was argued by Levy and Zhang et al. [14], and a discussion on CPP-MR values obtained by extrapolation will be presented in a forthcoming paper [15].

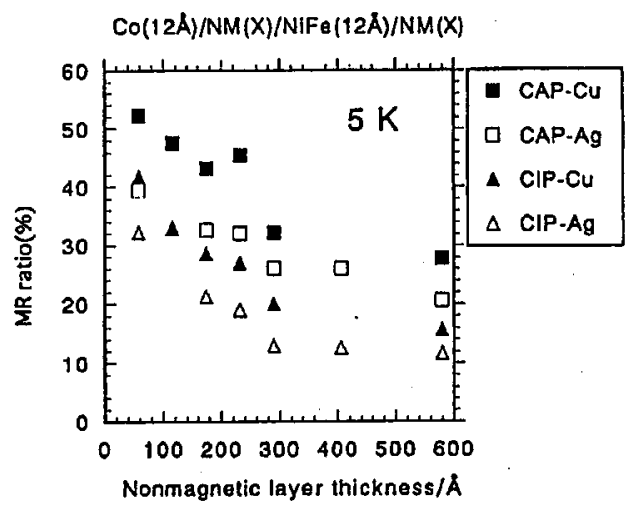

Fig. 5. CAP- and CIP-MR ratios at $5 \mathrm{~K}$ for $[\mathrm{Co}(12 \AA) / \mathrm{NM}(\mathrm{X}) / \mathrm{NiFe}(12 \AA) / \mathrm{NM}(\mathrm{X})]$, where $\mathrm{NM}$ is $\mathrm{Cu}$ or $\mathrm{Ag}$.

As non-magnetic spacer materials in the multilayer samples for CAP-MR measurements, $\mathrm{Cu}$ and $\mathrm{Ag}$ were adopted in the present experiment. The MR ratios at $5 \mathrm{~K}$ are plotted as a function of spacer layer thickness in Fig. 5. The results for $\mathrm{Cu}$ and $\mathrm{Ag}$ are qualitatively similar to each other. The MR ratios decrease with an increase in spacer layer thickness and CAP-MR ratios are always larger than CIP. The ratio of CAP-MR/CIP-MR has a tendency to increase with an increase in spacer layer thickness but the crrors are too large to analyze the relation quantitatively. The MR ratios for the Cu-based samples are in general larger than those for the Ag-based ones. This result suggests that $\mathrm{Cu}$ is a better choice a spacer to obtain a larger MR ratio. The spin dependent scattering probability at $\mathrm{Cu}$ interfaces seems to be larger than that at Ag interfaces. However, MR ratios depend also very much on crystallographic sample qualities. For the arguments on intrinsic physical parameters, spin dependent scattering probability for example, further experiments with improved sample qualities are required.

V-groove substrates were utilized also by Gijs et al. [16]. They deposited 
multilayers in a direction perpendicular to the side wall of the groove (see Fig. 3e). Below and on the top of the multilayers, well conducting $\mathrm{Cu}$ layers were deposited to give good contacts between multilayers in each groove and measurements on CPP-like MR were carried out. If multilayers deposited in a tilted direction are isolated with each other, as illustrated in Fig. 3f, magnetic systems with a wire are obtained [17].

\section{Magnetic multilayer wires}

Magnetic multilayers with a wire shape are prepared by depositing films from a tilted direction, perpendicularly to the (111) side planes of V-groove. For the study of magnetic viscosity, a five layer sample was prepared with the following structure: $[\mathrm{NiFe}(100 \AA) / \mathrm{Cu}(100 \AA) / \mathrm{NiFe}(10 \AA) / \mathrm{Cu}(100 \AA) / \mathrm{NiFe}(100 \AA)]$, which is a kind of a non-coupled type GMR system, similar to the spin-valve. If the period of $\mathrm{V}$-groove is $0.5 \mu \mathrm{m}$, the wire width of the deposited multilayer is $0.3 \mu \mathrm{m}$. Because of the wire shape, the magnetic layers have a uniaxial magnetic anisotropy along the groove direction. The magnetizations are therefore oriented along the groove. Separately, NiFe single layer wires with the same width but different thicknesses were prepared and the coercive forces of $\mathrm{NiFe}$ single layer wires were measured. The coercive forces were found to be fairly large, because of the wire shape, and to increase with an increase in thickness, in the single domain region, namely smaller than about $500 \AA$. In the five layer sample, since each magnetic layer is separated by $100 \AA \mathrm{Cu}$ layers, magnetization reversals occur independently. The thinner layer $(10 \AA \mathrm{NiFe})$ has a smaller coercive force and changes the direction of magnetization at a smaller field. In the magnetization curve shown in Fig. 6a, a small change is observed around $80 \mathrm{Oe}$, which corresponds to the magnetization reversal of the $10 \AA \mathrm{NiFe}$ layer. Since the magnetizations of $100 \AA \mathrm{NiFe}$ layers are much dominant, it is hard to draw a reliable information on the magnetic behaviour of the $10 \AA \mathrm{NiFe}$ layer from this magnetization measurement. In contrast, as shown in Fig. $6 \mathrm{~b}$, the MR curve at $5 \mathrm{~K}$ clearly indicates the magnetization reversal of the $10 \AA$ layer. Around $80 \mathrm{Oe}$, the $10 \AA$ layer changes the direction of magnetization, while those of the $100 \AA$ layers are invariant. Because of the antiparallel alignment of magnetizations, the MR curve shows a sharp increase, due to the GMR effect. With a more increase of external field, the direction of magnetization in $100 \AA$ layers gradually changes and the resistivity is slowly going down. Using this MR change, it is possible to estimate sensitively the degree of reversal of the $10 \AA \mathrm{NiFe}$ layer's magnetization.

In a wire-shape structure, magnetization reversals should take place by domain wall motions. If the magnetization becomes viscid, magnetization should be time dependent. The time dependence of the $10 \AA$ layer's magnetization was monitored by observing the MR in the field of $80 \mathrm{Oe}$, after applying an external field of 5000 Oe in the opposite direction. At low temperatures, the magnetization becomes viscid and the resistivity shows a time dependence. In the present experiment, about 4000 pieces of microwires were measured in parallel and the measured resistivity corresponds to the sum of magnetizations in each wire. Domain walls in each wire may move independently perhaps in some steps. From resistivity measurements, we are able to estimate the degree of magnetization reversal. If the 


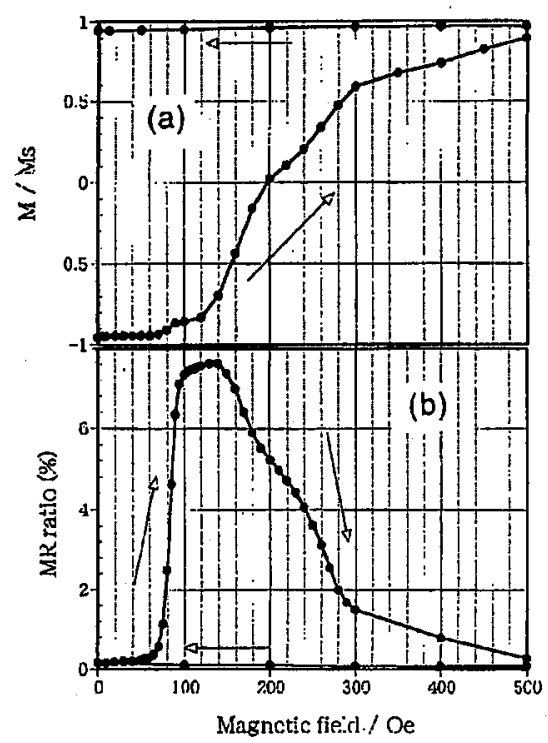

Fig. 6. (a) Magnetization curve at $5 \mathrm{~K}$ for a sample of multilayer wires, the structure is written in the text; (b) MR curve at $5 \mathrm{~K}$ for the same sample.

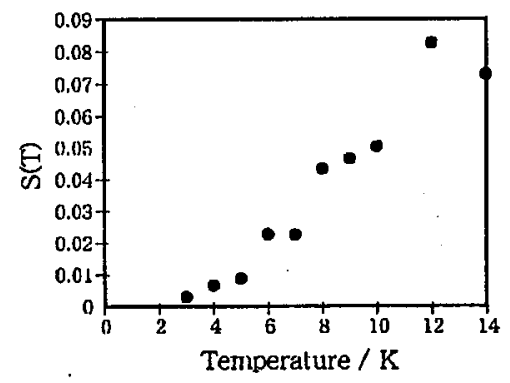

Fig. 7. Magnetic viscosity of $\mathrm{NiFe}$ wire (10 $\AA$ thick and $0.3 \mu \mathrm{m}$ width) as a function of temperature, estimated from time of $\mathrm{MR}$ at each temperature. $S(T)$ is magnetic viscosity at temperature $T$.

resistivity is time dependent, the viscosity is estimated. At low temperatures, the resistivity showed a slight but continuous increase. The resistivity values were then plotted as a function of $\log t$, where $t$ means time, and the magnetic viscosity was obtained as a coefficient of $\log t$. The time dependence of magnetization was measured at various temperatures and the magnetic viscosity was estimated by this method. The viscosity as a function of temperature, $S(T)$, is shown in Fig. 7. The result indicates a decrease in magnetic viscosity with a decrease in temperature. This is a reasonable result if a thermal activation process is a dominant mechanism for magnetization reversals.

According to recent theoretical investigations and some experimental results 
[18-21], there should exist a macroscopic quantum tunneling process (MQT) is small magnetic systems at low temperatures. Then, the viscosity should be temperature independent. The result in Fig. 7 is apparently temperature dependent, suggesting the thermal process is still dominant at this temperature region. To confirm the existence of MQT, more experiments at lower temperatures are necessary.

Although the results described here are in a primitive stage, it is proved that the wire-shaped samples prepared using microstructured substrates are useful for the study of mesoscopic magnetic systems. A very sensitive estimation of magnetization is possible from resistivity measurements on a specially designed multilayer using the principle of GMR.

\section{Acknowledgments}

The authors thank Drs. N. Hosoito and K. Mibu for discussion. The present work was supported by the Grant-in-Aid for developmental scientific research from Monbusho.

\section{References}

[1] Y. Otani, B. Pannetier, J.P. Nozieres, D. Givord, J. Magn. Magn. Mater. 126, 622 (1993).

[2] I. Nakatani, IEEE Trans. Magn., in press.

[3] N. Bardou, B. Bartenlian, F. Rousseaux, D. Decanini, F. Carcenac, E. Cambril, M.F. Ravet, C. Chappert, P. Veillet, P. Beauvillain, R. Megy, W. Geerts, J. Ferre, J. Magn. Magn. Mater. 156, 139 (1996).

[4] E. Bassous, IEEE Trans. ED 25, 1178 (1978).

[5] M.N. Baibich, J.M. Broto, A. Fert, F. Nguyen Van Dau, F. Petroff, P. Etienne, G. Creuzet, A. Friederich, J. Chazelas, Phys. Rev. Lett. 61, 2472 (1988).

[6] D.F. Mosca, A. Berthelemy, F. Petroff, A. Fert, P.A. Schroeer, W.P. Pratt Jr., R. Laloee, J. Magn. Magn. Mater. 94, L1 (1991).

[7] S.S.P. Parkin, Z.G. Li, D.J. Smith, Appl. Phys. Lett. 58, 2472 (1991).

[8] T. Shinjo, H. Yamamoto, J. Phys. Soc. Jpn. 59, 3061 (1990).

[9] B. Dieny, V.S. Speriosu, S.S.P. Parkin, B.A. Gurney, D.R. Wilhoit, D. Mauri, Phys. Rev. B 43, 1297 (1991).

[10] W.P. Pratt Jr., S.F. Lee, J.M. Slaughter, R. Laloee, P.A. Schroeder, J. Bass, Phys. Rev. Lett. 66, 3060 (1991).

[11] M.A.M. Gijs, S.K. Lenczowski, J.B. Giesbers, Phys. Rev. Lett. 70, 3343 (1993).

[12] L. Piraux, J.M. George, J.F. Despres, C. Leroy, E. Ferain, R. Legras; Appl. Phys. Lett. 65, 2484 (1994).

[13] Preliminary results on the $\mathrm{Cu}$-based samples were already reported: T. Ono, T. Shinjo, J. Phys. Soc. Jpn. 64, 363 (1995).

[14] P.M. Levy, S. Zhang, T. Ono, T. Shinjo, Phys. Rev. B 52, 16049 (1995).

[15] T. Ono, T. Shinjo, in preparation.

[16] M.A.M. Gijs, M.T. Johnson, A. Reinders, P.E. Huisman, R.J.M. van de Veerdonk, S.K.J. Lenczewski, R.M.J. van Gansewinkel, Appl. Phys. Lett. 66, 18 (1995). 
[17] A preliminary report was already submitted: T. Ono, Y. Sugita, T. Shinjo, J. Phys. Soc. Jpn. 65, (1996), in press.

[18] E.M. Chundnovsky, L. Gunther, Phys. Rev. Lett. 60, 661 (1988).

[19] B. Barbara, E.M. Chudnovsky, Phys. Lett. 145, 205 (1990).

[20] G. Tatara, H. Fukuyama, J. Phys. Soc. Jpn. 63, 2538 (1994).

[21] N. Giordano, J.D. Monnier, Physica B 194-196, 1009 (1994). 